\title{
NT-3 Replacement with Brain-Derived Neurotrophic Factor Redirects Vestibular Nerve Fibers to the Cochlea
}

\author{
Lino Tessarollo, ${ }^{1}$ Vincenzo Coppola, ${ }^{1}$ and Bernd Fritzsch ${ }^{2}$ \\ ${ }^{1}$ Neural Development Group, Mouse Cancer Genetics Program, National Cancer Institute, Frederick, Maryland 21701, and ${ }^{2}$ Department of Biomedical \\ Sciences, Creighton University, Omaha, Nebraska 68178
}

\begin{abstract}
Survival of inner ear sensory neurons depends on two neurotrophins, BDNF and NT-3, and their respective receptors, TrkB and TrkC. Because both receptors are present in the same neuron, it has been suggested that BDNF and NT-3 are functionally redundant in promoting neuronal survival. Knock-in of one ligand into the locus of the other one confirmed this hypothesis for the cochlea, leaving open the question of why two neurotrophins are required for proper innervation of the mammalian ear. Here, we show that the precise spatiotemporal pattern of expression of the two neurotrophins is essential for proper patterning of the inner ear innervation. Mice expressing BDNF under the control of the NT-3 promoter develop exuberant projections of vestibular sensory neurons to the basal turn of the cochlea. This projection can be enhanced by combining the transgene with a null mutation of BDNF. However, vestibular fibers rerouted into the cochlea do not reach hair cells and remain outside the organ of Corti, suggesting a chemotactic role for neurotrophins on these fibers. Our data provide genetic evidence that neurotrophins in the ear exert both survival and axon guidance roles.
\end{abstract}

Key words: cochlea; ear; guidance; neurotrophic; neurotropic; vestibular

\section{Introduction}

The inner ear of vertebrates is composed of three different sensory systems dedicated to the perception of sound, gravity, and various head movements (Lewis et al., 1985). Keeping the information gathered by these different systems distinct requires that connections of the ear with the brain develop such that each of the six end organs communicates its information selectively to specific areas of the brain for sensory information processing (Maklad and Fritzsch, 2003b; Newlands and Perachio, 2003; Ryugo and Parks, 2003). Therefore, connections of the cochlea have to develop so that specific areas are mapped in a precise topological manner onto auditory nuclei and higher-auditory centers (Rubel and Fritzsch, 2002) to ensure appropriate development of the tonotopic neuronal network that decodes direction-specific information (Kapfer et al., 2002). Recent evidence on the distribution of sensory neurons connecting various end organs suggests that matching sensory neurons to specific epithelia and central targets may not rely on mechanisms known from other sensory systems (Maklad and Fritzsch, 2003a). At the moment, it is fair to say that the molecular basis for the development of specific inner ear connections is still unknown (Fritzsch, 2003).

One aspect unique to the development of the mammalian ear is a complicated spatiotemporal pattern of expression of two neurotrophins, BDNF and NT-3 (Ernfors et al., 1992; Pirvola et al., 1992; Farinas et al., 2001) and an overlapping distribution of their

Received Dec. 15, 2003; revised Jan. 20, 2004; accepted Jan. 22, 2004

This work was supported by Grant R01 DC005590 (B.F.) from the National Institute on Deafness and Other Communication Disorders.

Correspondence should be addressed to Dr. B. Fritzsch, Department of Biomedical Sciences, Creighton University, Omaha, NE 68178. E-mail: Fritzsch@Creighton.edu.

DOI:10.1523/JNEUROSCI.5514-03.2004

Copyright $\odot 2004$ Society for Neuroscience $\quad$ 0270-6474/04/242575-10\$15.00/0 specific receptors, TrkB and TrkC (Ernfors et al., 1992; Ylikoski et al., 1993; Farinas et al., 2001). What exactly the function of two neurotrophins is in the mammalian ear remains somewhat unclear. Initial data suggested a hair cell-specific projection (Ernfors et al., 1995). Closer analyses of single null neurotrophin mutants showed that the developmental dynamics of neurotrophin expression leads to specific loss of sensory neurons to parts or entire sensory organs in which the other neurotrophin cannot compensate for its absence (Bianchi et al., 1996; Fritzsch et al., 1997). It has been suggested that the two neurotrophins of the ear may be functionally replaceable because their specific function appears to be related to their respective expression profile rather than any specifc signaling capacity (Farinas et al., 2001). Indeed, recent studies using replacement of either neurotrophin by the other indicates functional equivalence of either neurotrophin for the cochlea (Coppola et al., 2001; Agerman et al., 2003). Interestingly, although replacement of NT-3 with BDNF rescues the survival of the cochlea basal turn sensory neurons, lost in NT-3 null mutant mice, it also generates an exuberant innervation of the basal turn (Coppola et al., 2001).

Using this mouse model (Coppola et al., 2001), we now provide evidence that the delayed expression of BDNF in the basal turn of the cochlea is crucial to avoid redirection of vestibular fibers into the cochlea to generate an exuberant innervation. We also provide evidence that in the ear neurotrophins are not necessary for initial pathfinding. Together, these findings suggest that in the ear there are two distinct molecular guidance phases that control the pattern of innervation.

\section{Materials and Methods}

Mice. NT- $3^{\operatorname{tgBDNF}-1-}$ is a homozygous null mutation for the NT-3 gene that expresses BDNF under control of the NT-3 promoter. The 
NT- $3^{\text {tgBDNF }}$ knock-in mice, as well as the BDNF-deficient mice used in this study, were as described previously (Liebl et al., 2000; Coppola et al., 2001). Both NT-3 ${ }^{\operatorname{tgBDNF}}$ and BDNF mutant mice were backcrossed in a C57Bl/6 background, respectively, for 10 and 15 generations. Double and most of the single mutants were obtained from intercrosses of NT-3 3 tgBDNF/ BDNF double heterozygous mice. Timed embryos were obtained by overnight mating of doubly heterozygotes. The noon when the vaginal plug was observed was considered embryonic day (E) 0.5. Gravid uteri were removed from timed pregnant females at different stages of gestation $(\mathrm{E} 11.5,12.5,13.5)$, and embryos were dissected and fixed overnight in $4 \%$ paraformaldehyde (PFA) and $0.1 \mathrm{~m}$ phosphate buffer, $\mathrm{pH}$ 7.2. Anesthetized neonate animals were perfused with $4 \%$ PFA for retrograde labeling experiments.

All animals were treated in accordance with the guidelines provided by the Animal Care and Use Committee of the National Cancer Institute at Frederick, Maryland.

Analysis of inner ear innervation. We have analyzed a total of 10 ears from E11.5 mutants, 16 ears from E12.5 mutants, 24 ears from E13.5 mutants, and 54 newborn mutant ears. Among these ears were a total of 14 doubly null mutant ears, which we compared with 18 single BDNF null mutants and 18 single NT-3 ${ }^{\text {tgBDNF}}$ null mutants at E11.5, 12.5, and 13.5 and postnatal day (P) 0 (Table 1). Analysis consisted of labeling the afferent fibers to the ear from the brainstem and cerebellum using differently colored lipophilic tracers, PTIR 271 and PTIR 278 (Fritzsch et al., 2002). After insertion of the dyesoaked filter strips in the ear, the brainstem, or the cerebellum (rhombomere 1 during early embryonic development), the fixed heads were incubated for 2 or $4 \mathrm{~d}$ at $36^{\circ} \mathrm{C}$ until the dye had diffused into the fine terminals. The inner ears were then dissected, and ganglia and sensory epithelia were mounted flat in glycerol. Images were captured using a cooled CCD camera. In addition, ears were analyzed by confocal microscopy (Radiance 2000; Bio-Rad, Hercules, CA). Image stacks of flat mounted cochlea and vestibular end organs were taken and collapsed in the $z$-axis to reveal the entire pattern of innervation.

Brains were dissected, embedded in gelatin, and sectioned at $100 \mu \mathrm{m}$ as described previously (Maklad and Fritzsch, 2002). Mounted sections were analyzed using confocal microscopy as described above and previously (Maklad and Fritzsch, 2003a).

\section{Results}

We previously showed that loss of cochlear innervation caused by NT-3 deficiency can be rescued by BDNF expression under the NT-3 promoter (Coppola et al., 2001; Fritzsch et al., 2004). Paradoxically, we have also observed an extensive outgrowth of fibers selectively to the basal turn of the cochlea, despite some minor spiral ganglion neuron loss. Therefore, we have investigated whether these additional fibers came from sources other than the cochlea-specific spiral ganglion neurons. We focused on the vestibular afferents because only these ear afferents can reach the cerebellum and, thus, can readily be distinguished in retrograde labeling experiments (Dino et al., 2001; Barmack, 2003; Maklad and Fritzsch, 2003a; Newlands and Perachio,
Table 1. Number of ears per genotype analyzed at various stages

\begin{tabular}{|c|c|c|c|c|c|}
\hline Genotype & E11.5 & E12.5 & $\mathrm{E} 13.5$ & PO & Total \\
\hline WT & 4 & 4 & 4 & 8 & 20 \\
\hline NT $-3^{\operatorname{tgBDNF}-1+}$ & 2 & 2 & 6 & 12 & 22 \\
\hline NT-3 $3^{\operatorname{tgBDNF}-1-}$ & 2 & 4 & 8 & 8 & 18 \\
\hline NT- $3^{\operatorname{tgBDNF}+/-} / \mathrm{BDNF}^{+/-}$ & 6 & 10 & 10 & 12 & 38 \\
\hline NT- $3^{\text {tgBDNF-1- }} /$ BDNF $^{+/-}$ & 0 & 2 & 6 & 14 & 22 \\
\hline NT- $3^{\text {tgBDNF-l- }}{ }^{\text {BDNNF }}{ }^{-1-}$ & 2 & 2 & 2 & 8 & 14 \\
\hline NT- $3^{\operatorname{tgBDNF}+/-} / \mathrm{BDNF}^{-/-}$ & 2 & 4 & 6 & 16 & 28 \\
\hline BDNF $^{-1-}$ & 4 & 4 & 2 & 8 & 18 \\
\hline $\mathrm{BDNF}^{+1-}$ & 4 & 8 & 6 & 6 & 24 \\
\hline Total & 26 & 40 & 46 & 92 & 204 \\
\hline
\end{tabular}

WT, Wild type.
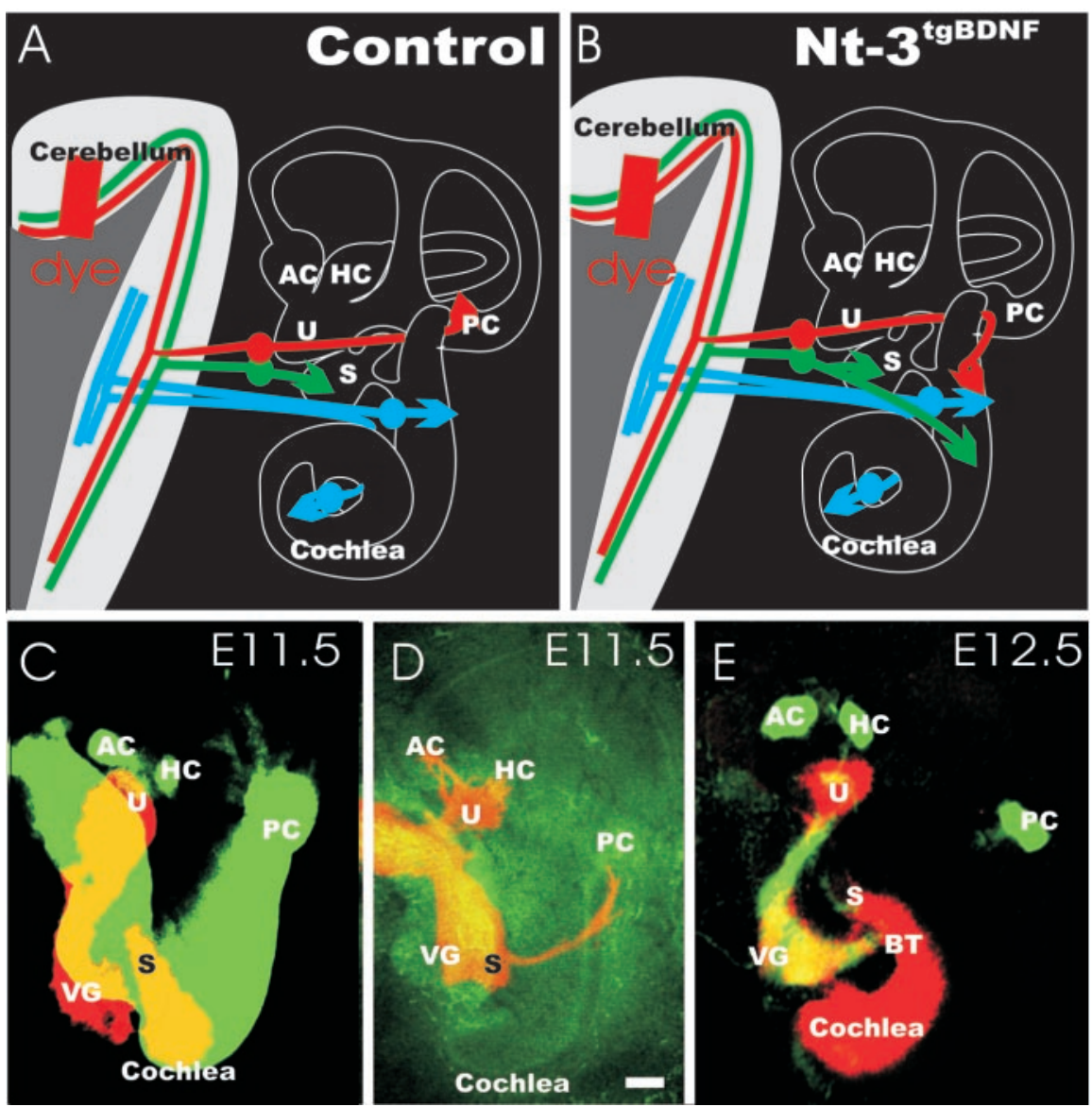

Figure 1. Schematic representation of the distribution of cochlear (blue) and vestibular (red and green) afferent fibers to the auditory and vestibular nuclei in a wild-type and a NT-3 ${ }^{\text {tgBDNF }}$ mutant mouse at $\mathrm{PO}$. $A, B$, Vestibular afferents from the saccule (green) and the posterior canal crista (red) project next to the cochlear nuclei to the vestibular nuclei of the brainstem but also reach the cerebellum. Injection of dye into the cerebellum, thus, labels only fibers to the vestibular sensory epithelia in wild-type animals $(A)$. However, in NT-3 $3^{\text {tgBNNF}}$ it shows redirected vestibular fibers to the cochlea $(B)$. $C-E$, Dynamic alteration in neurotrophin expression during early embryonic development relative to the initial fiber outgrowth. BDNF (false colored green) and NT-3 (false colored red) expression is shown at E11.5 ( $C$ and E12.5 $(E)$. Note that fibers extending to the posterior crista from the vestibular ganglion $(D)$ have to cross the basal turn of the cochlea that expresses NT-3 but not BDNF $(E)$. We hypothesize that expression of BDNF under NT-3 promoter control will result in redirection of posterior crista fibers because they have to cross areas that express BDNF in the transgenic animals. AC, Anterior crista; $H C$, horizontal crista; $P C$, posterior crista; $S$, saccule; U, utricle; VG, vestibular ganglion; BT, basal turn of the cochlea. Scale bar, $100 \mu \mathrm{m}$.

2003). Because central expression of neurotrophins is upregulated only after initial fiber projections are established (Fritzsch, 2003), we reasoned that only peripheral projection of sensory neurons might be profoundly affected by the misexpression of BDNF under the control of the NT-3 promoter (NT- $3^{\text {tgBDNF}}$ ) (Fig. 1). 


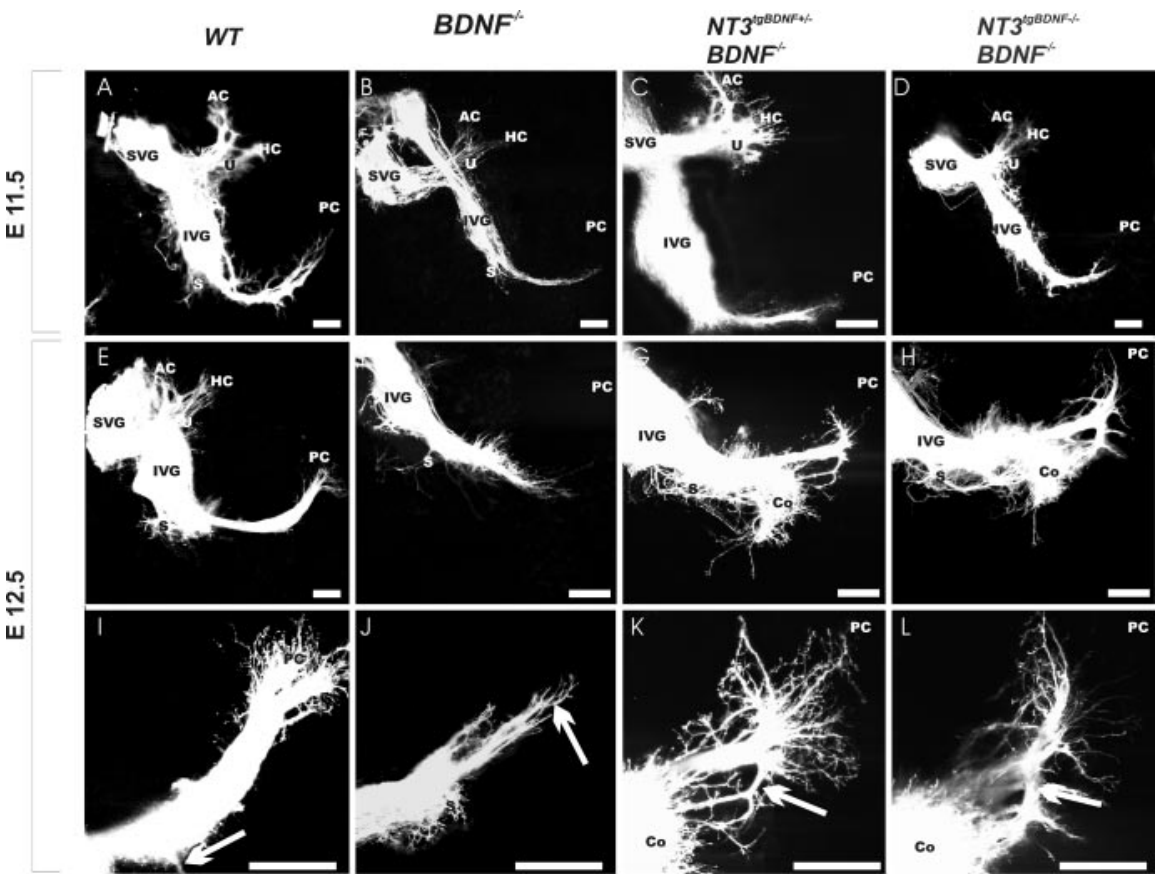

Figure 2. Various combinations of NT-3 ${ }^{\mathrm{tgBDNF}}$ and BDNF null mutations have limited effects on the pattern of inner ear innervation in early development compared with wild-type littermates $(A, E, I)$. At E11.5, the overall pattern of innervation of mutant mice with different combinations of NT-3 ${ }^{\text {tgBDNF}}$ and BDNF null mutations $(B-D)$ closely resembles that of wild type $(A)$. Only a reduction of fiber growth to the horizontal $(\mathrm{HC})$, anterior $(A C)$, and posterior $(P C)$ crista is observed when an endogenous BDNF level is eliminated $(B)$, even in the presence of NT-3 ${ }^{\text {tgBDNF}}$ expression (compare $A$ and $($ and $D$ ). At E12.5, transgenic expression of BDNF starts to cause qualitative differences on inner ear innervation of mutant animals relative to that of controls $(E-L)$. BDNF null mutants show no fibers extending to the posterior crista $(F, J)$ even if combined with NT- $3^{\operatorname{tgBDNF}}(G, H, K, L)$. Instead, these fibers are randomly oriented $(K)$ or directed toward the cochlea $(K, L$, arrow). There is an obvious growth of fibers to the cochlea $(G, H)$ in NT-3 ${ }^{\text {tgBDNF}}$ combined with BDNF null mutation. No such fibers are present in wild-type animals at this stage $(E)$. There is an apparent dosage effect on the degree of branch formation of the posterior crista fibers to the basal turn of the cochlea $(I, J$, arrow), with the furthest extension of fibers in the simple BDNF null mutants. Scale bar, $100 \mu \mathrm{m}$.

\section{Developmental day E11.5}

Analysis of inner ear innervation at E11.5 overall did not reveal striking differences between wild-type or mutant mice with the specific neurotrophin mutation combinations used in this study (Fig. $2 A-D$ ). Injections into rhombomere 1 at E11.5 when postmitotic neurons initiate fiber outgrowth showed only one occasional qualitative difference and less fiber outgrowth in the BDNF null mutant mice (Fig. $2 \mathrm{~B}$ ). Combining the BDNF null mutation with NT-3 ${ }^{\text {tgBDNF}}$ in heterozygosis or homozygosis resulted only in three of seven ears in fibers extending beyond the horizontal crista toward the posterior crista (data not shown). Even combining absence of BDNF under its own promoter with exclusive expression of BDNF under the NT-3 promoter resulted only in limited alterations in the branching pattern of the fiber growth to the posterior crista (Fig. 2C,D). Thus, initial vestibular fiber growth toward the developing sensory epithelia is not qualitatively affected by either complete absence of BDNF or misexpression of BDNF under NT-3 promoter control with or without BDNF under its own promoter control (Fig. 2C,D). These data suggest that at this stage neurotrophins do not exert a chemotropic function on inner ear afferent outgrowth but may, nevertheless, have some limited trophic effects as indicated by the reduced size of the ganglia (Fig. 2, compare $A, B$ ).

\section{Developmental day E12.5}

At E12.5, wild-type and mutant mice with various combinations of transgenic BDNF expression and endogenous BDNF null mutations showed obvious differences (Fig. $2 E-L$ ), especially in the innervation of the posterior crista (Fig $2 I-L)$ and the vestibular fiber growth to the basal turn of the cochlea (Fig. $2 E-H$ ). BDNF null mutant mice showed few fibers extending to, but none reaching, the posterior crista (Fig. 2F,J). Most interesting is the more profound outgrowth of fibers to the cochlea in mice with BDNF replacing NT-3 (Fig. 2G,H), a feature never seen in wild-type littermates or in younger transgenic animals (Fig. 2D,E). Brainstem injections showed only a few labeled spiral sensory neurons at this stage in wild-type mice (Fig. 2E) or simple transgenic heterozygotes (data not shown). Wild-type and NT3- ${ }^{\operatorname{tgBDNF}}$ heterozygous animals have an occasional growth of a few fibers from the posterior canal crista toward the cochlea (Fig. 2I, arrow). However, with the increased expression of the transgenic $\mathrm{BDNF}$ and concomitant reduction of the endogenous BDNF, more fibers extend toward the cochlea (Fig. $2 K, L$, arrow). In BDNF null mice with NT3- ${ }^{\text {tgBDNF}}$ in homozygosis or heterozygosis, no fibers actually reach the posterior crista epithelia (Fig. 2G, $H, K, L)$. Instead, in these mice fibers stall between the basal turn of the cochlea and the posterior crista and either extend in multiple directions or toward the basal turn of the cochlea (Fig. $2 K, L$ ). In one animal, all fibers of the posterior crista twig turn to the cochlea (data not shown).

Taken together, these data suggest that initial neurite outgrowth is mainly BDNF independent. However, the topology of the BDNF expression exerts some control over the sites to which fibers are going to project as early as E12.5. Variation of the qualitative pattern in a group of animals with a specific genotype is much less than the variation between different genotypes, suggesting that the variations observed reflect the genotype differences and not random variations not associated with the genotype.

\section{Developmental day E13.5}

Injection of tracers into the cerebellum of wild-type animals reveals no fibers to the cochlea but fibers to all five vestibular sensory epithelia (Fig. 3A). BDNF null mutants at E13.5 have only an occasional residual fiber projecting toward the posterior canal crista (Fig. 3D), as described previously (Bianchi et al., 1996). Furthermore, only a single fiber was sometimes found extending from the utricle to the anterior and horizontal crista (Fig. 3C). Most importantly, when lipophilic tracers were injected into the cerebellum, neither BDNF null mice nor wild-type littermates showed more than an occasional fiber projecting to the cochlea (Fig. $3 A, D$ ). In contrast, even NT-3 tgBDNF heterozygotic embryos showed rerouting of fibers to the basal turn of the cochlea (Fig. $3 F$ ), which was even more profound in NT-3 tgBDNF homozygotic embryos (Fig. 3B). Such rerouting occurred in both homozygous and heterozygous animals and originated predominantly from the saccule (Fig. 3, compare $A, B$ ) with fibers also rerouting to the cochlea from the nerve twig to the posterior canal (Fig. 3B, arrow).

When NT-3 ${ }^{\text {tgBDNF}}$ was combined with the BDNF null muta- 
tion, more fibers were rerouted from the vestibular epithelia into the cochlea (Fig. $3 F$, arrowhead). Furthermore, many more fibers could be traced to the anterior and horizontal crista (Fig. 3E) than in the simple BDNF null mutants (Fig. 3C). In addition, fibers to the posterior canal could be traced toward the posterior crista but appeared to stall between the basal turn of the cochlea and the posterior canal, radiating parallel to the cochlea (Fig. $3 F$, arrow). No projections entered the posterior canal, but a sizable bundle was invariably found to extend toward the basal turn of the cochlea (Fig. 3F, arrow). These data suggest a preferred branch node near the posterior crista at which fibers switch from a non-neurotrophin-dependent mode to a neurotrophin-dependent mode of navigation, neurite survival, or both.

\section{Inner ear innervation at birth}

We next analyzed the effects of NT$3^{\mathrm{tgBDNF}}$ in the presence or absence of endogenous BDNF on the innervation of the vestibular epithelia at birth (P0) (Fig. 4). Previously, we have shown that NT$3^{\text {tgBDNF}}$ rescues almost completely the innervation of the cochlea basal turn, which is entirely lost in the NT-3 null mutants (Fritzsch et al., 1997). This result had suggested that BDNF and NT-3 are function-

ally equivalent for the survival of cochlear neurons (Coppola et al., 2001). A more recent study has confirmed our findings on the cochlea by replacing endogenous BDNF with NT-3. This study also suggested that NT-3 cannot rescue vestibular fibers (Agerman et al., 2003). As reported previously (Bianchi et al., 1996), we found that BDNF null mutants have a reduced, although still relatively dense, innervation of the utricle and very few fibers, if any, that extend to the anterior and horizontal crista and none to the posterior crista (Fig. $4 F$ ) (data not shown). The anterior and horizontal cristae of NT-3 ${ }^{\text {tgBDNF}}$ mice are always innervated, independently of the level of endogenous BDNF (wild type, heterozygous, or null) (Fig. $4 A, C$, inset). However, all NT-3 ${ }^{\text {tgBDNF }}$ transgenic mice had projections of fibers outside the sensory epithelia radiating beyond the cristae (Fig. $4 A, C, D$, arrowheads) or to an area devoid of sensory hair cells near the utriculo-saccular foramen (Figs. 4A-E, arrows). The central projections of these extensions appear to be vestibular like, because these fibers are labeled from the cerebellum injections of dye (Fig. $4 G$, arrow) despite the fact that they are not in touch with hair cells in the ear. Previous analysis of NT-3 expression with an NT-3-LacZ reporter mouse line showed that there is a faint NT-3 presence in those areas of fiber extension (Farinas et al., 2001). These data suggest that NT- $3^{\text {tgBDNF}}$ expression can rescue fibers to the anterior and horizontal crista but not to the posterior crista. This rescue might be related to the close proximity of the anterior and horizontal crista to the utricle, which could provide a source of diffusible BDNF. However, maintenance of posterior crista fibers would require BDNF to diffuse from the cochlea that is a far more distant target. In addition, NT-3 ${ }^{\text {tgBDNF}}$ causes fiber rerouting into areas that have no sensory hair cells but express limited amounts of NT-3, in the transgenic animals replaced by BDNF. Together, these data support the notion that areas of NT-3 expression in the nonsensory part of the ear will attract and support fibers even in the absence of hair cells, provided they produce BDNF.

We next examined why the posterior crista fibers could not be rescued. NT- $3^{\text {tgBDNF}}$ can rescue the innervation loss of the basal turn of the cochlea caused by NT-3 deletion (Coppola et al., 2001). However, many more fibers project to the cochlea in these animals compared with controls. Surprisingly, cerebellar dye injections label fibers that extend toward the basal turn of the cochlea. These fibers are present in all NT-3 $3^{\text {tgBDNF}}$ animals examined, but their density varies depending on the mouse genotype of the endogenous BDNF (Fig. 5B-D). Only a few fibers can be labeled from the cerebellum in the doubly heterozygous NT$3^{\text {tgBDNF }}$ BDNF animals (Fig. $5 A$ ) and none in wild-type animals (Fig. 5, inset). Furthermore, these fibers innervate exclusively the basal turn of the cochlea and arborize near both inner and outer hair cells (Fig. 5A). Careful preparation of the posterior cristae nerve together with the basal turn of the cochlea shows that many of these fibers derive from the posterior crista nerve (Fig. $5 B$, arrow). Combining NT $-3^{\text {tgBDNF}}$ homozygosity with a reduction (Fig. 5C) or loss (Fig. 5D) of endogenous BDNF shows a dosedependent effect with $\mathrm{NT}-3^{\mathrm{tgBDNF}^{-1-}}$; $\mathrm{BDNF}^{-/-}$mice having the most vestibular fiber projections to the basal turn of the cochlea (Fig. $5 B-D$ ). Interestingly, these mice also show a robust projection to both the inner and, in particular, the outer hair cell regions (Fig. 5D). These data suggest that $\mathrm{NT}-3^{\mathrm{tgBDNF}-1-}$; $\mathrm{BDNF}^{-1-}$ animals lack posterior crista innervation because surviving posterior crista nerve fibers are rerouted into the basal turn of the cochlea. Furthermore, the rerouting suggests that the significant expression of BDNF under the NT-3 promoter control in the basal turn of the cochlea may provide a source of BDNF that 

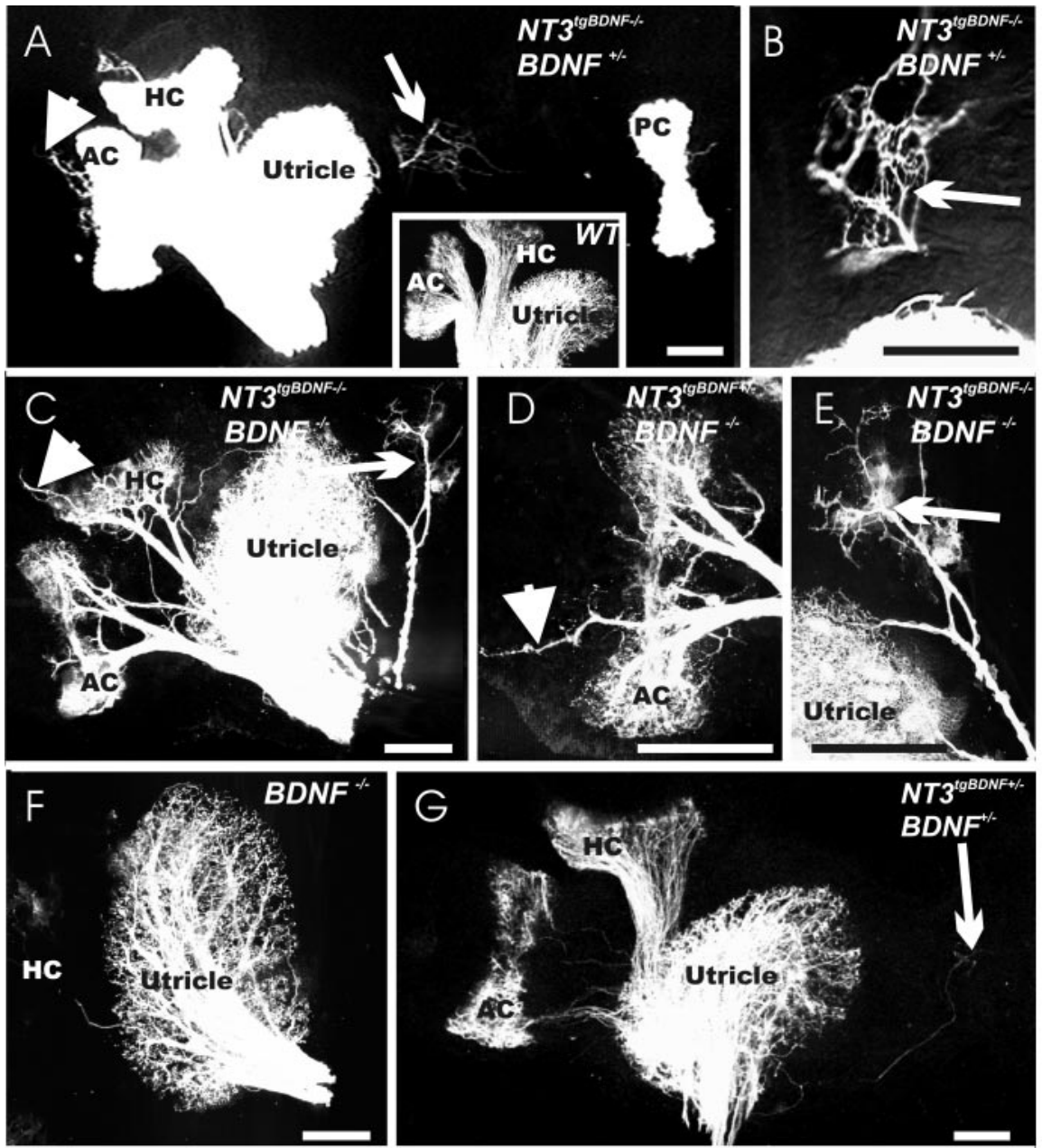

Figure 4. BDNF exerts both survival and chemoatractant roles on fibers innervating the vestibular epithelia. At PO, in the otherwise normally innervated vestibular epithelia of double NT-3 ${ }^{\mathrm{tgBDNF}} / \mathrm{BDNF}$ heterozygous animals, some fibers labeled from the brainstem extend beyond the utricle ( $A$, arrow; higher magnification in $B$ ) and cristae sensory epithelia (arrowhead) into areas known to express limited amounts of NT-3. No such fibers exist in wild-type animals ( $A$, inset). A simple BDNF null mutation results in loss of fibers to the posterior crista (data not shown) and a reduced innervation of the utricle $(F)$. Combining the BDNF null mutation with NT3 $3^{\text {tgBDNF }}$ homozygosity causes a more profound innervation of the utricle $(C)$ but also a substantial rescue of fibers to the anterior and horizontal crista $(C, D)$. As observed in $A$, transgenic expression of BDNF under NT-3 promoter control causes vestibular fibers to extend beyond the sensory epithelia in areas devoid of hair cells ( $C-E$, arrows and arrowheads; $E$ is a higher magnification of () . Fibers innervating these areas devoid of hair cells seem to be able to develop a rather normal projection into the brain $(A-E)$, because they can be labeled from the cerebellum $(G)$ much like in wild-type animals ( $A$, inset). $A C$, Anterior crista; HC, horizontal crista; PC, posterior crista. Scale bar, $100 \mu \mathrm{m}$.

by far exceeds the limited and delayed amounts of endogenous BDNF synthesized in the nonsensory epithelium near the posterior crista in the NT- ${ }^{\text {tgBDNF-1- }}$; $\mathrm{BDNF}^{-/-}$. Thus, the fibers rerouted in the transgenic animals appear to follow concentration gradients of BDNF, which are critical for chemoattracting these fibers to their precise target (Farinas et al., 2001).

Taken together, these data suggest that vestibular fibers of the posterior crista and possibly the saccule are rerouted in NT$3^{\text {tgBDNF }}$ transgenic animals to project to the basal turn of the cochlea. To further prove this point, we next injected one lipophilic tracer into the posterior crista and a differently colored tracer (Fritzsch et al., 2002) into the basal turn of the cochlea. For technical reasons, we used NT-3 ${ }^{\text {tgBDNF}}$ mutants combined with BDNF in heterozygosis because these animals have robust basal turn projections as well as a significant innervation to the posterior crista (data not shown). Injections of a red-colored dye into the cochlea and of a green-colored dye into the posterior crista showed an overlapping distribution of vestibular sensory neurons in the inferior vestibular ganglion (Fig. 6A-C) adjacent to the cochlea nerve. The position of the posterior crista neurons in the inferior vestibular ganglion was exactly as described previously (Maklad and Fritzsch, 2002, 2003a), suggesting that indeed all rerouted afferents to the cochlea derive from neurons that project to the posterior crista and the saccule in wild-type animals. Incidentally, a small and variable number of double-labeled vestibular neurons were also found, and their existence was directly demonstrated using single-dye injection into the cochlea and posterior crista, respectively. In three of three animals with a cochlear injection, we found labeled fibers to the posterior crista as well as some fibers projecting toward the utricle and possibly to the saccule (Fig. $6 D, E$ ). Furthermore, injection of a different colored dye into the posterior crista could trace in three of three animals fibers to the basal turn of the cochlea (Fig. $6 \mathrm{~F}$ ). These fibers showed bidirectional projections below outer and inner hair cells. We also examined the brainstem projections in two animals in which we performed simultaneous dye applications into the cochlea and the posterior crista. Whereas control animals showed no fibers entering through the vestibular root, NT-3 ${ }^{\text {tgBDNF}}$ animals revealed fibers entering through the vestibular root and projections to the vestibular nuclei that overlap completely with fibers labeled from the posterior crista (Fig. 6G,H, yellow fibers). Some fibers were found to extend from the dorsal cochlear nucleus (Fig. $6 \mathrm{H}$ ) into the vestibular nuclei. However, such fibers were also occasionally found in control animals (data not shown). In summary, this analysis confirmed that indeed the vestibular fibers of the posterior crista are rerouted to project to the basal turn of the cochlea in NT- $3^{\text {tgBDNF}}$ transgenic animals while maintaining their central projection as vestibular fibers.

We next analyzed in more detail the terminations of the vestibular fibers in the cochlea by using whole mounted and sectioned ears. Fibers labeled in the cochlea from dye placed in the cerebellum (Fig. 7) showed terminal morphologies unknown from either afferent or efferent fibers at this stage of development (Bruce et al., 1997; Fritzsch et al., 1997). In fact, individual fibers formed multiple branches near the outer or inner hair cells or extended for some distance along the inner or outer hair cells (Fig. 7A-C). These data suggest that BDNF, in addition to its chemoattractant role on these sensory fibers, can also influence their branching pattern and morphology of the terminals.

To further investigate how the altered BDNF expression can affect the pattern of innervation of inner and outer cochlear hair cells, we first looked at the effects caused by the simple BDNF and NT-3 deletions. As reported previously (Bianchi et al., 1996), we 
found that BDNF null mutants have abundant afferent fibers projecting to inner hair cells in the basal turn, whereas they have a reduced presence of fibers to several rows of the outer hair cells (Fig. 7G) resembling closely the basal turn innervation in wildtype littermates (Fig. 7E). In contrast, NT-3 null mutant mice retain some innervation of inner hair cells with only occasional fibers to one row of the outer hair cells (Fig. 7D). These data confirm our previous findings that outer hair cell innervation in the base depends predominantly on NT-3 with some minor effects mediated by BDNF (Fig. 7D, G). Filling afferent fibers from the cerebellum of BDNF mutants combined with the NT-3 tgBDNF mutation showed a more prominent projection of fibers to the outer hair cells (Fig. $7 F$ ) despite the fact that no NT-3 is present in these mice. Surprisingly, most of the vestibular fibers from the cerebellum do not reach the level of the cochlear hair cells. Rather, they terminate underneath the cochlea hair cells just below the basilar membrane near the spiral vessels (Fig. $7 F$ ). We next analyzed the fiber organization in sectioned material. Radial sections through the cochlea show unique fibers around the habenula perforate that do project only rarely toward the hair cells of the organ of Corti (Figs. 7 H,I). These data suggest that the habenula perforate is more than just a hole through which spiral ganglion afferents enter to the organ of Corti. In fact, the apparent inability of vestibular fibers to enter the organ of Corti suggests that the habenula perforate may serve as an additional filter to allow spiral ganglion neuron afferents, but not vestibular ganglion neuron afferents, to enter the organ of Corti.

In summary, our data provide compel-

ling evidence that BDNF expressed under the control of the NT-3 promoter can rescue not only cochlear (Coppola et al., 2001), but also vestibular, neurons in BDNF null mutant mice. However, vestibular fibers, in addition to being rescued, are also rerouted to unusual targets near the vestibular epithelia and to the cochlea, where BDNF is expressed under NT-3 promoter control.

\section{Discussion}

Mammalian neurotrophins and their receptors are crucial for sensory neuron survival (Mocchetti, 2001). In contrast to this function, much less is known about their suggested role in pathfinding (Ming et al., 2002). Recently, using beads soaked with neurotrophins, fiber outgrowth toward the ectopic neurotrophin site has been reported in vivo (Tucker et al., 2001). However, while providing support for an axon guidance role for neurotrophin, these data do not distinguish between a neurotrophic and a neurotropic function of neurotrophins. By using the inner ear as a model system and a gene replacement strategy, we have provided for the first time conclusive evidence suggesting that neurotrophins can exert both roles on the same neuron in vivo. Sen- sory neurons of the inner ear have an overlapping distribution of the neurotrophin receptors TrkB and TrkC (Ylikoski et al., 1993; Farinas et al., 2001) that allows to support their survival with either BDNF or NT-3 (Coppola et al., 2001; Agerman et al., 2003). By replacing NT-3 with BDNF in combination with different endogenous levels of BDNF, we have shown that the initial afferent fiber outgrowth in the ear is independent of neurotrophins because peripheral dendritic growth is unperturbed by any genetic manipulation of neurotrophin expression. In a second phase of fiber outgrowth, starting at E12.5, neurotrophins not only support neuronal survival but exert also a role in dendrite guidance because mis-expression of BDNF causes rerouting of vestibular neuron dendrites to the cochlea.

Thus, after an initial phase of normal fiber outgrowth, which is neurotrophin independent, the precise spatiotemporal expression of neurotrophins is indeed crucial to avoid developmental rerouting of vestibular fibers to the cochlea of the ear. These data provide evidence that support the existence of two distinct phases in molecular guidance in the developmental pattern of innervation of the inner ear. Similar support of BDNF for dendritic 

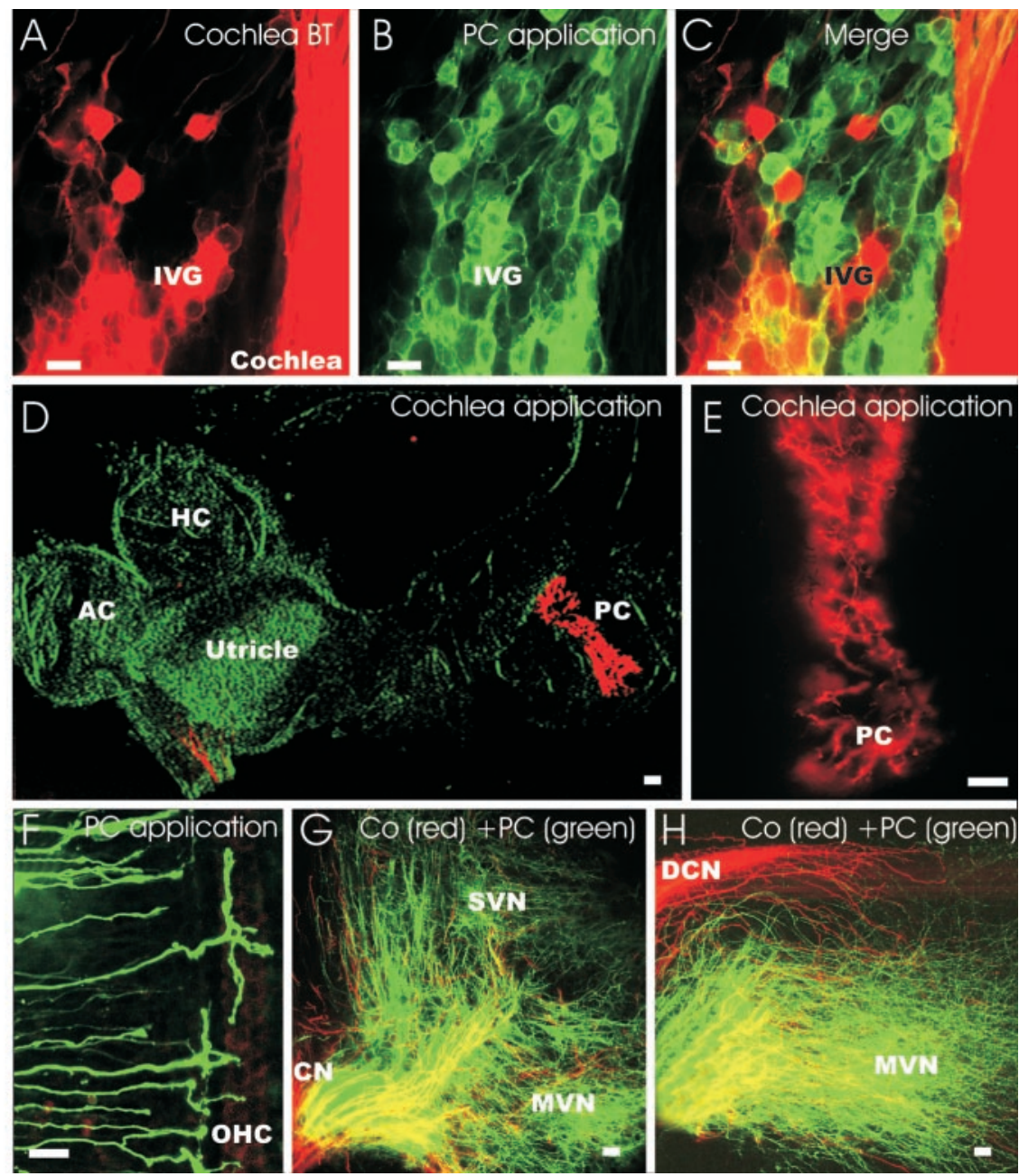

Figure 6. NT-3 ${ }^{\operatorname{tgBDNF}}$ and BDNF doubly heterozygous mice have vestibular fibers projecting to both the posterior crista and the cochlea. Injecting two differently colored lipophilic tracers into the cochlea basal turn $(A$, red) and the posterior crista $(B$, green) shows that these cells overlap in the inferior vestibular ganglion ( $C$. A small and variable number of vestibular neurons (between 3 and 27 double-labeled neurons; $n=3$ ears) have branches to both the cochlea and the posterior crista ( $C$, yellow cells). This bifurcation is directly demonstrated by the labeling of neurons that project to the posterior crista when dye application is in the cochlea $(D, E)$ and labeling of fibers projecting to the cochlea is visualized by dye application from the posterior crista $(F)$. Examination of the vestibular system after application of the red dye into the cochlea reveals labeling of many fibers to the posterior crista and a few fibers to the saccule and utricle $(D, E)$. Likewise, injection of green dye into the posterior crista labels fibers to the basal turn of the cochlea $(F)$. Central projections after application of a red dye to the cochlea and a green dye to the posterior canal crista shows green fibers to the medial and superior vestibular nuclei and red fibers to the auditory nuclei $(G, H)$. However, some red fibers enter the vestibular root and extend into the vestibular nuclei $(G, H$, yellow). In contrast, only red fibers reach the dorsal cochlear nucleus $(H)$ and the ventral cochlear nucleus $(G)$. IVG, Inferior vestibular ganglion; $P C$, posterior crista; MVN, medial vestibular nucleus; SVN, superior vestibular nucleus; $C N$, ventral cochlear nucleus; DCN, dorsal cochlear nucleus. Scale bar: $A-D, G, H, 10 \mu \mathrm{m} ; E, F, 100 \mu \mathrm{m}$.

branching and maintenance, but not initial growth, has been reported for forebrain pyramidal neuron dendrites (Gorski et al., $2003 \mathrm{~b}$ ) and supports the involvement of BDNF and its receptor in neuronal plasticity (Minichiello et al., 1999; Gorski et al., 2003a) as well as in pathological learning deficits (Chen et al., 2003; Martinowich et al., 2003).

\section{The role of neurotrophins in fiber guidance}

We have previously shown that undifferentiated hair cells (Xiang et al., 2003) or even complete elimination of the sensory epithelium, a site of neurotrophin expression (Pauley et al., 2003), is compatible with directed fiber growth. Recently, it has been reported that semaphorins may, at least in part, provide molecular guidance at this stage because manipulating the neuropilin-1 re- ceptor causes fiber misguidance ( $\mathrm{Gu}$ et al., 2003). The profound effect caused by manipulations in the neurotrophin system, after the initial growth toward the sensory epithelia has occurred, indicates that neurotrophins mediate survival and routing of fibers only in a second phase. It is tempting to refer to this redirection as a neurotrophic fiber guidance because it coincides with the survival of sensory neurons.

We have found that in NT-3 $3^{\operatorname{tgBDNF}}$ mice fibers project to both vestibular sensory epithelia as well as to nonsensory areas known to express NT-3 in wild-type mice (Farinas et al., 2001). For example, the exogenous BDNF can support fibers innervating the anterior and horizontal crista as well as small nonsensory patches near the utricle. Such fibers form both in the presence or absence of endogenous BDNF (Fig. 4). These data suggest that BDNF per se is a potent chemoattractant that support fibers at least as well as hair cells and may be the major attractor of fiber growth to hair cells because it is prominently expressed in all developing hair cells (Pirvola et al., 1992; Farinas et al., 2001).

\section{The pattern of experimentally rerouted fibers resembles patterns in nonmammalian vertebrates}

In nonmammalian vertebrates, fibers can project to the basilar papilla (cochlea) along two different pathways (Fritzsch, 1987, 2002; Fritzsch and Wake, 1988). Interestingly, in those vertebrates, fibers to the cochlea that come off the nerve twig to the posterior crista seem to branch off at or near the branch point of fibers to the papilla neglecta, similarly to what we observed here in the NT- $3^{\text {tgBDNF}}$ mice (Figs. $3 F, 8)$. The effect caused by neurotrophin replacement on these fibers when they reach this area suggest that, at this position, they may shift to a different mode of guidance (e.g., from semaphorins to neurotrophins).

Mammals seem to be the only vertebrates in which the ear expresses an additional neurotrophin, NT-3, in significant amounts (Pirvola et al., 1997; Hallbook et al., 1998). Our previous data have shown that expression of NT-3 in the basal, high-frequency turn of the cochlea of mammals is followed by a delayed upregulation of BDNF in this turn (Farinas et al., 2001). This seems to provide a unique solution to the rerouting problem of fibers from the posterior crista nerve twig found in some nonmammalian vertebrates. However, premature expression of BDNF under NT-3 promoter control in the basal turn of the NT-3 $3^{\text {tgBDNF}}$ transgenic mice experimentally nullifies this solution, causing fibers to reroute in a pattern similar to that of some nonmammalian vertebrates (Fritzsch and Wake, 1988). More data on the relative timing of hair cell proliferation and 
expression of BDNF in nonmammalian vertebrates is needed to critically test this hypothesis.

\section{The role of neurotrophins in intraepithelial innervation}

Although these data settle the functional role for neurotrophins in interepithelial connection specification, a second role in intraepithelial connection specification is still controversial. It has been suggested that inner hair cell innervation of the cochlea is lost in NT-3 and TrkC null mutants, whereas outer hair cell innervation is lost in BDNF and TrkB null mutants (Ernfors et al., 1995; Schimmang et al., 1995). This idea is consistent with the fact that the number of spiral sensory neurons lost in a given neurotrophin null mutant closely matches the number of a given type of spiral sensory neurons (Fritzsch et al., 2001; Agerman et al., 2003). However, additional detailed analysis of $\mathrm{NT}-3^{-1-}$ mice (Fritzsch et al., 1997) showed that inner hair cells, and only few outer hair cells, (Fig. 7D) are innervated. The BDNF null mutant mice studied here have afferent fibers innervating several rows of outer hair cells (Fig. 7G), as previously reported for other mutant lines (Bianchi et al., 1996; Agerman et al., 2003). The profound growth of fibers to outer hair cells in the NT- $3^{\text {tgBDNF}}$ mice is not compatible with a cell-specific attraction of different spiral afferents.

\section{BDNF mediates spatiotemporal distribution and direction of cochlear fibers}

The situation is more complex for the fibers innervating the cochlea. For one thing, NT-3 expression progresses along the cochlea (from base to apex), across the cochlea (from lesser to greater epithelial ridge), and changes cell types (from Deiter's cells to inner hair cells (Farinas et al., 2001). In contrast to some vestibular epithelia, fibers enter the cochlea in both TrkB and BDNF null mice (Fritzsch et al., 1995; Schimmang et al., 1995; Bianchi et al., 1996; Agerman et al., 2003). However, replacement of NT-3 with BDNF, while rerouting vestibular fibers to the cochlea, is not sufficient to allow these fibers to cross the habenula perforate. Only occasional branches can reach the inner hair cells (Fig. 7). It is possible that the habenula perforata may actually provide a selective and attractive entry point for spiral ganglion neuron dendrites into the cochlea and that the limited diffusion of BDNF from its expression in supporting cells may provide neurotrophic support for these fibers. Alternatively, cochlear hair cells may release a non-neurotrophin factor that acts as a specific attractor for spiral sensory afferents and to which vestibular sensory afferents cannot respond. Previous work has shown that cochlear in-
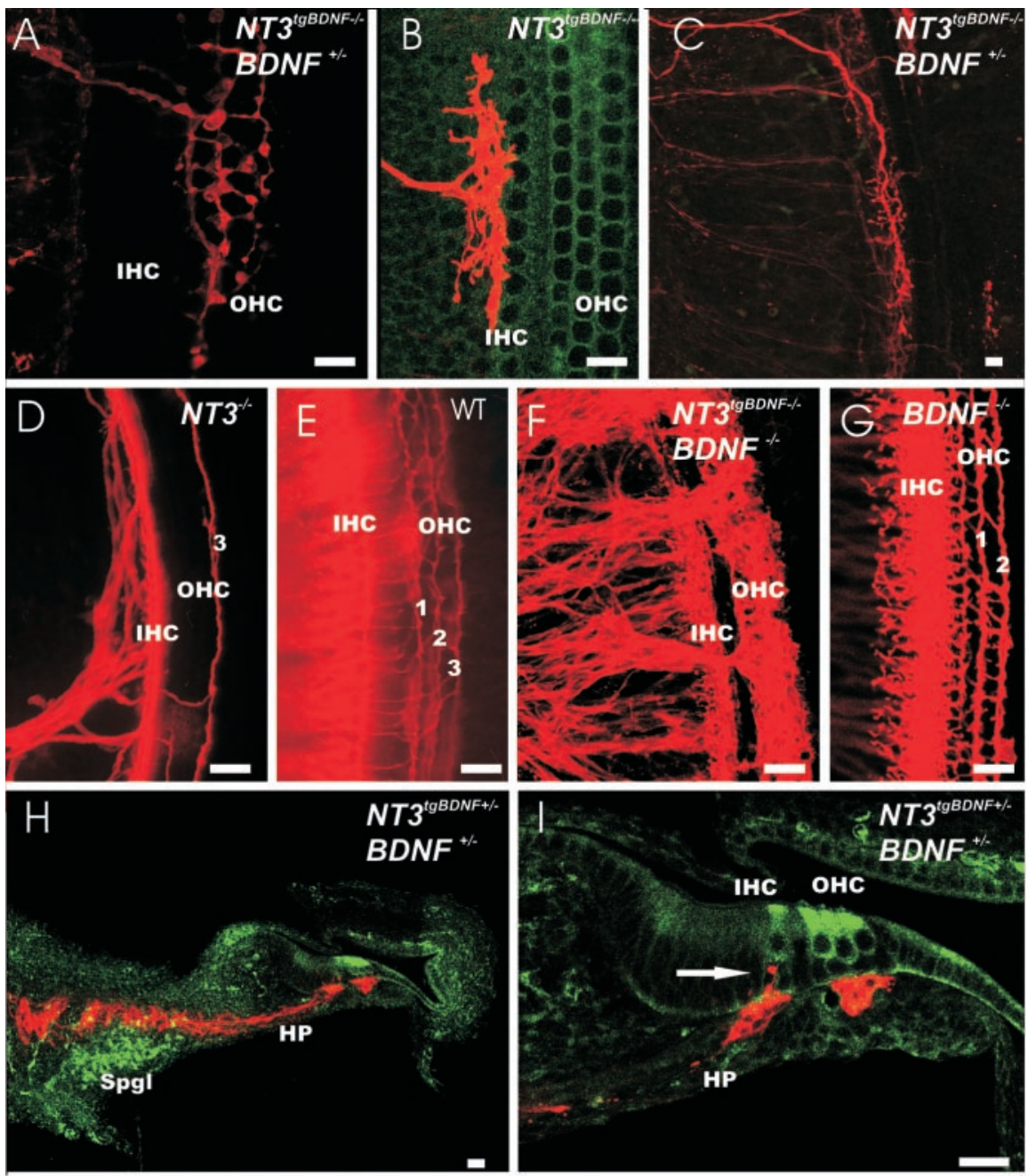

Figure 7. BDNF influences the branching pattern and morphology of fibers innervating the inner and outer hair cells of the cochlea. $A-C$, Pattern of single fibers near the hair cells. D-G, Densities of innervation of inner and outer hair cells in the cochlea of wild-type $(E)$ or various neurotrophin $(D, F, G)$ mutants. $H, I$, Pathway of fibers filled from the cerebellum to the hair cells of the cochlea. Whole mounted ears are all oriented with the base pointing up. Single fibers show bifurcation with either equal distance growing toward the basal and the apical turn $(A, B)$ or show an extensive course near the inner or outer hair cells in either apical (C) or basal direction (data not shown). In NT-3 null mutants, the basal turn receives only innervation along the inner hair cells anal fiber going to one row $(3)$ of the outer hair cells $(D)$. As in wild-type mice $(E)$, fibers spiral from the apex to the base. BDNF null mutants in the base have fibers to inner hair cells and to at least two rows of outer hair cells $(1,2 ; G)$. In contrast Sections through the cochlea show that these fibers bypass the spiral ganglion and run through the habenula perforate but stay whereas no fiber passes through the basilar membrane to reach the outer hair cells. Spgl, Spiral ganglion; IHC, inner hair cells; $\mathrm{OHC}$, outer hair cells; HP, habenula perforata. Scale bar: $A, B, D-G, I, 10 \mu \mathrm{m} ; C, H, 100 \mu \mathrm{m}$.

nervation to the apex is rather normal in mice with limited differentiation of hair cells (Xiang et al., 2003).

In summary, we present here data on afferent development of the ear using the recently reported transgenic expression of BDNF under NT-3 promoter control (Coppola et al., 2001). Previous work has shown that BDNF and NT-3 can functionally replace each other in the cochlea (Coppola et al., 2001; Agerman et al., 2003). By combining the NT-3 ${ }^{\text {tgBDNF}}$ mice with BDNF null mutation, we show that the initial fiber growth is not determined by neurotrophins (Fig. 8). Additional fiber growth occurs during the cell death phase in such mutants (E12.5-E15.5), leading to a massive rerouting of vestibular sensory neuron afferents to the cochlea. This rerouting is mediated by BDNF, suggesting that 


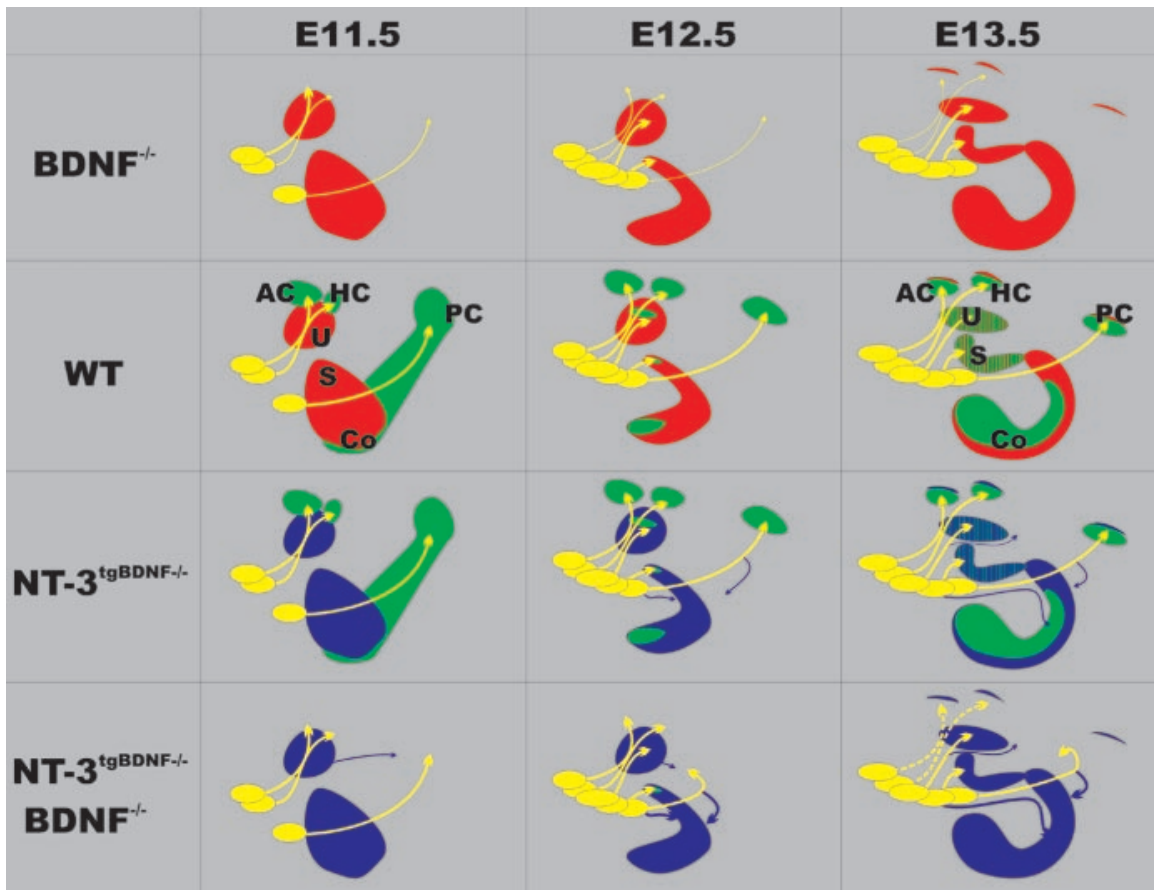

Figure 8. Schematic representation of the changes in the developmental pattern of vestibular innervation of the inner ear caused by the altered expression of BDNF and NT-3. BDNF and NT-3 expression are shown, respectively, in green and red; NT-3 $\operatorname{tgBDNF}$ is shown in blue. At E11.5, the initial fiber projection is predominantly to the anterior (AC), horizontal (HC), and posterior (PC) crista and overall is identical in all genotypes. At E12.5, fibers to the crista organs are reduced in BDNF null mutants, and by E13.5, there is a complete loss of projections to the posterior crista (top row). The late and limited expression of NT-3 next to the crista sensory epithelia at E13.5 (top right) seems to be unable to rescue those fibers. Starting at developmental day E12.5 there is a significant growth of fibers to the utricle (U) and saccule (S) in all genotypes. At this stage, homozygous NT-3 ${ }^{\text {tgBDNF}}$ animals show a progressive development of novel fiber tracts (shown in blue), which extend either from the vestibular ganglion directly to the cochlea ( $\mathrm{C} 0$ ) or emanate from the posterior crista twig (bottom right pictures). More of these fibers are found if the transgenic BDNF is combined with the BDNF null mutation (blue arrows in the two bottom rows). In addition, some fibers that do not reach or innervate the posterior crista reroute to the basal turn of the cochlea (bottom right).

neurite growth is primarily regulated in the ear by BDNF, whereas NT-3 acts predominantly as a survival factor (Fig. 8). This finding suggests that growth of afferents to regenerating hair cells (Kawamoto et al., 2003) may be primarily mediated by BDNF expression in differentiating hair cells.

\section{References}

Agerman K, Hjerling-Leffler J, Blanchard MP, Scarfone E, Canlon B, Nosrat C, Ernfors P (2003) BDNF gene replacement reveals multiple mechanisms for establishing neurotrophin specificity during sensory nervous system development. Development 130:1479-1491.

Barmack NH (2003) Central vestibular system: vestibular nuclei and posterior cerebellum. Brain Res Bull 60:511-541.

Bianchi LM, Conover JC, Fritzsch B, DeChiara T, Lindsay RM, Yancopoulos GD (1996) Degeneration of vestibular neurons in late embryogenesis of both heterozygous and homozygous BDNF null mutant mice. Development 122:1965-1973.

Bruce LL, Kingsley J, Nichols DH, Fritzsch B (1997) The development of vestibulocochlear efferents and cochlear afferents in mice. Int J Dev Neurosci 15:671-692.

Chen WG, Chang Q, Lin Y, Meissner A, West AE, Griffith EC, Jaenisch R, Greenberg ME (2003) Derepression of BDNF transcription involves calcium-dependent phosphorylation of MeCP2. Science 302:885-889.

Coppola V, Kucera J, Palko ME, Martinez-De Velasco J, Lyons WE, Fritzsch B, Tessarollo L (2001) Dissection of NT3 functions in vivo by gene replacement strategy. Development 128:4315-4327.

Dino MR, Perachio AA, Mugnaini E (2001) Cerebellar unipolar brush cells are targets of primary vestibular afferents: an experimental study in the gerbil. Exp Brain Res 140:162-170.

Ernfors P, Merlio JP, Persson H (1992) Cells expressing mRNA for neuro- trophins and their receptors during embryonic rat development. Eur J Neurosci 4:1140-1158.

Ernfors P, Van De Water T, Loring J, Jaenisch R (1995) Complementary roles of BDNF and NT-3 in vestibular and auditory development. Neuron 14:1153-1164.

Farinas I, Jones KR, Tessarollo L, Vigers AJ, Huang E, Kirstein M, de Caprona DC, Coppola V, Backus C, Reichardt LF, Fritzsch B (2001) Spatial shaping of cochlear innervation by temporally regulated neurotrophin expression. J Neurosci 21:6170-6180.

Fritzsch B (1987) The inner ear of the coelacanth fish Latimeria has tetrapod affinities. Nature 327:153-154.

Fritzsch B (1992) The water-to-land transition: evolution of the tetrapod basilar papilla, middle ear and auditory nuclei. In: The evolutionary biology of hearing (Webster DB, Fay RR, Popper AN, eds), pp 351-375. New York: Springer.

Fritzsch B (2003) Development of inner ear afferent connections: forming primary neurons and connecting them to the developing sensory epithelia. Brain Res Bull 60:423-433.

Fritzsch B, Wake MH (1988) The inner ear of gymnophione amphibians and its nerve supply: a comparative study of regressive events in a complex sensory system. Zoomorphology 108:210-217.

Fritzsch B, Silos-Santiago I, Smeyne R, Fagan AM, Barbacid M (1995) Reduction and loss of inner ear innervation in trkB and trkC receptor knockout mice: a whole mount DiI and scanning electron microscopic analysis. Audit Neurosci 1:401-417.

Fritzsch B, Farinas I, Reichardt LF (1997) Lack of neurotrophin 3 causes losses of both classes of spiral ganglion neurons in the cochlea in a regionspecific fashion. J Neurosci 17:6213-6225.

Fritzsch B, Silos-Santiago I, Farinas I, Jones K (2001) Neurotrophins and neurotrophin receptors involved in supporting afferent inner ear innervation. In: The neurotrophins (Mocchetti I, ed), pp 149-164. San Diego: Salzburger and Graham.

Fritzsch B, Muirhead KA, Gray B, Maklad A (2002) Diffusion and imaging properties of three new lipophilic tracers, PKH2, PKH26 and PTIR271, and their use for triple labeling of neuronal profiles. Soc Neurosci Abstr 519.1.

Fritzsch B, Tessarollo L, Coppola V, Reichardt LF (2004) Neurotrophins in the ear: their roles in sensory neuron survival and fiber guidance. Prog Brain Res 146:265-278.

Gorski JA, Balogh SA, Wehner JM, Jones KR (2003a) Learning deficits in forebrain-restricted brain-derived neurotrophic factor mutant mice. Neuroscience 121:341-354.

Gorski JA, Zeiler SR, Tamowski S, Jones KR (2003b) Brain-derived neurotrophic factor is required for the maintenance of cortical dendrites. J Neurosci 23:6856-6865.

Gu C, Rodriguez ER, Reimert DV, Shu T, Fritzsch B, Richards LJ, Kolodkin AL, Ginty DD (2003) Neuropilin-1 conveys semaphorin and VEGF signaling during neural and cardiovascular development. Dev Cell 5:45-57.

Hallbook F, Lundin LG, Kullander K (1998) Lampetra fluviatilis neurotrophin homolog, descendant of a neurotrophin ancestor, discloses the early molecular evolution of neurotrophins in the vertebrate subphylum. J Neurosci 18:8700-8711.

Kapfer C, Seidl AH, Schweizer H, Grothe B (2002) Experience-dependent refinement of inhibitory inputs to auditory coincidence-detector neurons. Nat Neurosci 5:247-253.

Kawamoto K, Ishimoto S, Minoda R, Brough DE, Raphael Y (2003) Math1 gene transfer generates new cochlear hair cells in mature guinea pigs in vivo. J Neurosci 23:4395-4400. 
Lewis ER, Leverenz EL, Bialek WS (1985) The vertebrate inner ear. Boca Raton, FL: CRC.

Liebl DJ, Klesse LJ, Tessarollo L, Wohlman T, Parada LF (2000) Loss of brain-derived neurotrophic factor-dependent neural crest-derived sensory neurons in neurotrophin- 4 mutant mice. Proc Natl Acad Sci USA 97:2297-2302.

Maklad A, Fritzsch B (2002) The developmental segregation of posterior crista and saccular vestibular fibers in mice: a carbocyanine tracer study using confocal microscopy. Dev Brain Res 135.

Maklad A, Fritzsch B (2003a) Partial segregation of posterior crista and saccular fibers to the nodulus and uvula of the cerebellum in mice, and its development. Brain Res Dev Brain Res 140:223-236.

Maklad A, Fritzsch B (2003b) Development of vestibular afferent projections into the hindbrain and their central targets. Brain Res Bull 60:497-510.

Martinowich K, Hattori D, Wu H, Fouse S, He F, Hu Y, Fan G, Sun YE (2003) DNA methylation-related chromatin remodeling in activity-dependent BDNF gene regulation. Science 302:890-893.

Ming GL, Wong ST, Henley J, Yuan XB, Song HJ, Spitzer NC, Poo MM (2002) Adaptation in the chemotactic guidance of nerve growth cones. Nature 417:411-418.

Minichiello L, Korte M, Wolfer D, Kuhn R, Unsicker K, Cestari V, RossiArnaud C, Lipp HP, Bonhoeffer T, Klein R (1999) Essential role for TrkB receptors in hippocampus-mediated learning. Neuron 24:401-414.

Mocchetti I (2001) The neurotrophins. San Diego: Salzburger and Graham.

Newlands SD, Perachio AA (2003) Central projections of the vestibular nerve: a review and single fiber study in the Mongolian gerbil. Brain Res Bull 60:475-495.
Pauley S, Wright TJ, Pirvola U, Ornitz D, Beisel K, Fritzsch B (2003) Expression and function of FGF10 in mammalian inner ear development. Dev Dyn 227:203-215.

Pirvola U, Ylikoski J, Palgi J, Lehtonen E, Arumae U, Saarma M (1992) Brain-derived neurotrophic factor and neurotrophin 3 mRNAs in the peripheral target fields of developing inner ear ganglia. Proc Natl Acad Sci USA 89:9915-9919.

Pirvola U, Hallbook F, Xing-Qun L, Virkkala J, Saarma M, Ylikoski J (1997) Expression of neurotrophins and Trk receptors in the developing, adult, and regenerating avian cochlea. J Neurobiol 33:1019-1033.

Rubel EW, Fritzsch B (2002) Auditory system development: primary auditory neurons and their targets. Annu Rev Neurosci 25:51-101.

Ryugo DK, Parks TN (2003) Primary innervation of the avian and mammalian cochlear nucleus. Brain Res Bull 60:435-456.

Schimmang T, Minichiello L, Vazquez E, San Jose I, Giraldez F, Klein R, Represa J (1995) Developing inner ear sensory neurons require TrkB and $\mathrm{TrkC}$ receptors for innervation of their peripheral targets. Development 121:3381-3389.

Tucker KL, Meyer M, Barde YA (2001) Neurotrophins are required for nerve growth during development. Nat Neurosci 4:29-37.

Xiang M, Maklad A, Pirvola U, Fritzsch B (2003) Brn3c null mutant mice show long-term, incomplete retention of some afferent inner ear innervation. BMC Neurosci 4:2.

Ylikoski J, Pirvola U, Moshnyakov M, Palgi J, Arumae U, Saarma M (1993) Expression patterns of neurotrophin and their receptor mRNAs in the rat inner ear. Hear Res 65:69-78. 